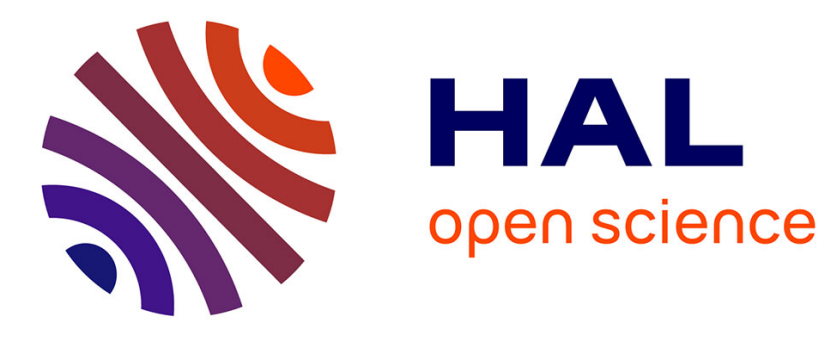

\title{
LPR: A Light Puncture Robot for CT and MRI Interventions
}

Ivan Bricault, Emilie Jauniaux, Nabil Zemiti, Céline Fouard, Elise Taillant, Frédéric Dorandeu, Philippe Cinquin

\section{- To cite this version:}

Ivan Bricault, Emilie Jauniaux, Nabil Zemiti, Céline Fouard, Elise Taillant, et al.. LPR: A Light Puncture Robot for CT and MRI Interventions. IEEE Engineering in Medicine and Biology Magazine, 2008, 27 (3), pp.42-50. 10.1109/EMB.2007.910262 . hal-00311442

HAL Id: hal-00311442

https://hal.science/hal-00311442

Submitted on 18 Aug 2008

HAL is a multi-disciplinary open access archive for the deposit and dissemination of scientific research documents, whether they are published or not. The documents may come from teaching and research institutions in France or abroad, or from public or private research centers.
L'archive ouverte pluridisciplinaire HAL, est destinée au dépôt et à la diffusion de documents scientifiques de niveau recherche, publiés ou non, émanant des établissements d'enseignement et de recherche français ou étrangers, des laboratoires publics ou privés. 


\title{
LPR: A Light Puncture Robot for CT and MRI Interventions
}

\author{
I. Bricault ${ }^{1,2}$, E. Jouniaux ${ }^{1}$, N. Zemiti ${ }^{1}$, C. Fouard ${ }^{1}$, E. Taillant ${ }^{1}$, \\ F. Dorandeu ${ }^{3}$, and Ph. Cinquin ${ }^{1, *}$
}

(1) TIMC-IMAG Laboratory - Computer Assisted Medical Interventions Team - IN3S - 38706 La Tronche Cedex - France

(2) Medical Imaging Department - University Hospital - 38043 Grenoble cedex - France

(3) Department of Toxicology - Centre de Recherche du Service de Santé des Armées - 38700 La Tronche - France

(*) Corresponding author: Ivan.Bricault@imag.fr

\begin{abstract}
This paper presents a new robotic architecture designed to perform abdominal and thoracic punctures under CT (Computer Tomography) or MRI (Magnetic Resonance Imaging) guidance for diagnostic or therapeutic purposes. Innovations concerning the robotic architecture, materials and energy sources are described. Segmentation and registration algorithms have been developed to localize the robot on images coming from CT or MRI devices, and a specific control loop is used to check the movements and the positioning of the robot. The results of the initial experiments that were made under CT and MRI environments are presented.
\end{abstract}

Keywords - Interventional radiology, MR compatibility, computer-assisted medical interventions (CAMI), localization, segmentation, registration.

\section{INTRODUCTION}

In the interventional radiology field, image guided percutaneous interventions are common procedures used for diagnostic or therapeutic purposes. Biopsies, abscess drainages or tumor ablations can be performed percutaneously. The clinical demand for such interventions is growing, since they are minimally invasive, and they are often able to prevent the need for a surgical intervention.

The first step in interventional radiology is the puncture of a target, which can be an anatomical structure or a tumor, and whose diameter is generally 1 centimeter or greater to be considered as clinically significant. These percutaneous punctures can be complicated procedures. The needle 
insertion is most often controlled with CT (Computed Tomography), but the planned trajectory can be particularly difficult to implement by the radiologist and to track on CT images when the needle is not aligned with a simple direction of the image plane. Indeed, tilted insertion paths are sometimes advised to avoid organs or ribs. Several attempts may be necessary to reach the target, causing a loss of time and more traumas than expected to the patient. Moreover, both radiologist and patient are exposed to X-rays when CT is used to guide, in real time, the puncture process.

Compared to CT, MRI is a promising non-irradiating imaging modality for the real-time control of a puncture, but specific constraints apply in a MRI environment, in particular because of the limited access to the patient allowed by traditional closed MR devices: the diameter of the bore does not exceed $60 \mathrm{~cm}$ which is also occupied by the patient.

The use of robots for surgical interventions is an approach that is now proven to increase the quality of operations and to establish new types of surgical procedures (see [1] for an up-to-date overview of this research field). In the context of interventional radiology, puncture robots may be very helpful. They have a potential to work within CT or MRI, and to decrease the intervention duration while providing optimal accuracy and safety to patients.

This has motivated our research for the development of a new lightweight robot for abdominal and thoracic percutaneous procedures, compatible with CT, open MR and closed MR. Our aim was to control in real-time the initial positioning of the needle and the puncture movement itself. Thus the robot had to be able to operate alone, but with remote medical supervision, inside the CT or MR guidance imaging device.

This robot (cf. Figure 1), named LPR (acronym for Light Puncture Robot), has an original compact body-supported architecture, which is naturally able to follow the patient body surface respiratory movements. It is entirely made of plastic, and uses MR-compatible pneumatic actuators 
powered by compressed air. It is localized via an image-based control using a localization device, totally integrated to the robot. The physician is also included in the control loop, since he/she selects the target and the entry point, and supervises the whole process.

\section{Related work}

Since the early 1990s more than 35 surgical robotic systems have been developed [1]. In the field of MRI interventional robotics, there exists to our knowledge only one commercial MRI compatible system: the Innomotion ${ }^{\mathrm{TM}}$ robot for percutaneous interventions (Innomedic, Germany, www.innomedic.de $)$.

Innomotion $^{\mathrm{TM}}$ is a pneumatic robot fully MR compatible developed to provide precise and reproducible needle positioning inside the magnet [2]. It consists of a 6 DoFs (Degrees of Freedom) robot arm which is attached to a $260^{\circ}$ arch mounted to the patient table and can be passively pre-positioned according to the region of interest. The robot position control is based on optical fiber position encoders. The kinematics of the device has been optimized for use in close MRI bore and CT scanners. However, the current version of the system provides only position guidance, i.e., the robot indicates to the physician the insertion point and the needle orientation. For closed bore MRI interventions, it is difficult to advance the needle manually inside the magnet bore. The patient table has to be moved outside the bore to perform the puncture correctly. However, if the robot is mounted to the table and not body supported by the patient (which is the case of the Innomotion ${ }^{\mathrm{TM}}$ system), any non-intentional movement of the patient during the table displacement may make change in the planned needle trajectory with respect to the desired one. Consequently, as we cannot have real time needle guidance outside the MR bore, such robot architecture is not robust with respect to patient movement and might cause injury to the patient when delicate organs are considered. 
Other MRI interventional robots are currently available at research laboratories. The major challenge for the development of these systems is the MR compatibility.

For MR compatibility questions the reader can refers to [3] where the MR compatibility of some materials using their magnetic susceptibility have been investigated, to [4] where the authors have proposed design criteria of mechatronic devices, and to [5] where simulations, and methods useful in predicting the magnetic and electromagnetic compatibility were suggested.

The following section provides an overview of research activities in the area of MRI interventional robotics.

Several research groups have developed MR compatible robots actuated by ultrasonic motors. Although these actuators are not affected by the magnetic field, their encasings contain conductive material that result in substantial image artefacts [6]. However, such actuators could be placed faraway from the MR magnet as in [7] and [8] where MRI compatible robots for biopsy and therapeutic interventions in the breast were presented and in [9] where the design of a novel MR compatible manipulator for prostate intervention was detailed. In [7], [8], and [9] the authors have shown that, for very specific clinical applications like interventions in the breast or prostate, it is possible to have long distance interaction between the control unit (ultrasonic motors) outside the MRI magnet and the patient inside the magnet. For such interventions, even if long mechanical connections are used, the robot configuration does not introduce notable flexion in the system.

In [10], a 5 DoFs surgical assistance robot is presented. It has been designed to aid minimally invasive surgical techniques under open low-field MRI scanners, such as positioning and directing a catheter or a laser pointer. All parts of the robot were made from paramagnetic materials such as titanium alloy and plastics. The mechanical body axes are located above the surgeon's head and are driven by linear non-magnetic ultrasonic motors placed outside the magnet bore. In 
this configuration, the magnetic susceptibility and the current of the motors have little impact on the MR compatibility. However, the main disadvantage of this configuration is that the long arms of the robot decrease the rigidity of the system. In addition, open low-field MRI scanners induce a problem of cost and availability, since open interventional MRI systems are rare and about twice more expensive than closed ones. Moreover, the image quality obtained using open lowfield MRI is not as good as the one obtained with closed MRI systems.

To summarize, the solution of using ultrasonic motors placed faraway from the MR magnet, combined with long mechanical transmissions is adapted only to very specific clinical objectives where the manipulator rigidity is guaranteed.

Authors in [11] have proposed a new approach to eliminate the ultrasonic motor's noise on the MR images. It consists in placing the system actuation (ultrasonic motors, motors controller and power supplies) in a Faraday cage, next to or inside the MR scanner. All wires and optical encoders were shielded and directed inside the Faraday cage. The experimental results show the shielding solution does not eliminate completely the noise but the image quality is sufficient for real-time imaging. The authors expect that better shielding would further decrease the noise.

Electrostatic motors could also be used within an MR environment. Compared to ultrasonic motors, they operate at lower frequencies (typically, up to $1 \mathrm{kHz}$ ) and at lower currents, so they should interfere less with MRI imaging [12]. However, as the electrostatic motor is driven by a three phase AC high voltage source ( $\simeq 2 \mathrm{kV}$ peak to peak), they still require developments on the power electronics to be used in the operation room.

Alternatively to ultrasonic or electrostatic motors, a hydrostatic transmission approach is proposed in [6] and a one DoF MRI and functional MRI compatible robot is presented. The concept is based on a tele-manipulator system with a mechanical transmission between the master and the slave. The master part of the robot (conventional actuator) that contains the interfering or sensi- 
tive components is placed outside the magnet shield, in the control room. A hydrostatic connection is used to transmit force and motion to an MR compatible slave, made from non-conducting, non-ferromagnetic polymers, placed next to or inside the MR scanner. For position control, the robot uses a home made fiber optic displacement sensor. The main feature of this system is that it is equipped with a force sensor, based on reflected light intensity measurement over optical fibers, that measures interaction forces with the human subject.

A new robot, named the MrBot [2], was recently developed at the John Hopkins University. It uses a new type of pneumatic step motors (the PneuStep) specifically designed for MRI application. The robot is customized for transperineal needle insertion for prostate gland under either open or closed bore MRI scanners. It is exclusively constructed of nonmagnetic and dielectric materials such as plastics, ceramics, and rubbers and is electricity free. Fiber optic encoding is used for position feedback, so that all electric components are distally located outside the MRI shield.

In [13], it is shown that devices constructed using binary polymer based actuators, called Electrostrictive Polymer Actuators (EPAM) are able to function within the MRI without degrading its imaging performance. The binary nature of these actuators eliminates the need for feedback sensors for motion control. Their construction is fundamentally inexpensive and simple. They can be constructed essentially from plastic, making their potential cost low enough to be disposable. This attractive approach remains to be explored further.

\section{The LPR architectural design: answers to CT/MRI compatibility and safety issues}

The Light Puncture Robot architecture is mainly based on the previous works done at the TIMC laboratory on the Tele-Echography Robot [14] and the Light Endoscopy Robot [15]. It is lightweight $(1 \mathrm{~kg})$ and compact $(15 \times 23 \mathrm{~cm})$. As shown in Figure 1, the main feature of this ro- 
bot is its original body supported architecture [16]. This provides an intrinsic compensation for some physiological (breathing) or accidental movements of the patient, since the robot follows patient's abdomen/thorax surface. Moreover, the installation of the system is particularly simple, so that the necessary set-up time is greatly reduced. A photo of the LPR robot is shown in Figure 2 .

This concept of placing the robot on the patient has been also chosen in [17] for endoscopic surgery (the $\mathrm{MC}^{2} \mathrm{E}$ robot), in [18] for percutaneous cholecystostomy interventions (the UMI system), and in [19] for CT-guided percutaneous interventions (the CT-Bot system).

The LPR possesses 5 DoFs (Degrees of Freedom) and is composed of two parts. The main part, the needle-holder that is laid directly on the patient's body, provides 3 DoFs (Figure 3): a translation $d \in\left[\begin{array}{ll}0 & 9 \mathrm{~cm}\end{array}\right]$ along the needle axis, a rotation $\theta \in\left[\begin{array}{ll}0 & 2 \pi\end{array}\right]$ w.r.t. (with respect to) the robot's platform and around an axis normal to the patient's body, and an inclination $\phi \in\left[\frac{-\pi}{18} \quad \frac{\pi}{3}\right]$ of the needle holder w.r.t. the robot's base.

The needle-holder puncture part (Figure 4) includes clamps used to grasp the needle, and a translation unit which is able to perform a fast puncture (above $9 \mathrm{~cm} / \mathrm{second}$ ) to perforate the skin or organs walls. To guarantee that the puncture movement will be straight, the needle is inserted through a guide (a small drilled ball) placed at the centre of the robot base. Once the needle movement has been achieved, clamps can release the needle and let it follow the movements of patient's target organ, thus avoiding injuries.

The puncture depth is controlled thanks to a thrust bearing (a stop) which position has to be specified by the physician during the path planning. If the target distance from the entry point is greater than the needle-holder stroke, the robot can perform a deep insertion by releasing the 
needle and grasping it higher. To perform this operation, the intervention of the physician is needed.

The second part of the robot is composed of a support frame and four actuators that provide the two remaining DoFs, i.e., the translation over the patient's body which gives natural stand and orientation to the robot (Figure 1 and Figure 2). This movement is done thanks to 4 straps bound to the needle-holder platform on one side and to the actuators on the other side. The architecture of the support frame of LPR offers the possibility to make a height adjustment of $10 \mathrm{~cm}$ to adapt the robot to patient's corpulence and to the imaging system size.

The LPR is plastic made (nylon, delrin, and epoxy) in order to ensure MR compatibility and a minimum of artefacts under CT environment. In order to be totally compatible with MR environment, compressed air is used as energy source. The robot controller and air compressor are linked to the actuators by $7 \mathrm{~m}$ long plastic tubes, allowing them to be outside the MR room, beyond the magnetic hazard zone.

A new and original way to use pneumatic actuators, based on clock making principles, was developed. Each actuator is composed of two sets of pistons and cylinders, one for each movement direction. On each side of a cylinder, one air inlet brings the compressed air (4 bar pressure) to the piston. Air is alternatively injected in each compartment. The first step consists in making the piston moving and pushing the associated sprocket wheel by one increment; the second step consists in making the piston going back without any action on the wheel thanks to the use of a passive joint (Figure 5). Note that this incremental movement is possible only in one direction, thus permitting to block the robot in a certain position.

A worm is assembled to the sprocket wheel axis and works with its corresponding gear (Figure 6 and Figure 7). For translation, the actuators have a pulley that allows the straps to be entan- 
gled/disentangled (Figure 7). The actuators can also be disengaged in order to perform a manual pre-positioning of the needle holder when the robot is installed on the patient.

Finally, this method allows a very easily and precisely controlled movement of the different parts of the robot. The overall robot specifications are summarised in Table 1.

Table 1: Robot specifications

\begin{tabular}{|c|c|}
\hline Description & value \\
\hline Air pressure & $4 \mathrm{bar}$ \\
\hline Actuators max. frequency & $3 \mathrm{~Hz}$ \\
\hline $\begin{array}{c}\text { Translational resolution } \\
\text { (patient body plan ) }\end{array}$ & $0.1414 \mathrm{~mm} /$ impulse \\
\hline $\begin{array}{c}\text { Needle penetration resolu- } \\
\text { tion }\end{array}$ & $0.15625 \mathrm{~mm} /$ impulse \\
\hline Base rotation resolution & $0.3^{\circ} / \mathrm{impulse}$ \\
\hline $\begin{array}{c}\text { Needle orientation resolu- } \\
\text { tion }\end{array}$ & $0.375^{\circ} / \mathrm{impulse}$ \\
\hline $\begin{array}{c}\text { Base rotation range } \\
\text { Needle orientation range }\end{array}$ & $\phi \in\left[\begin{array}{ll}\frac{-\pi}{18} & \frac{\pi}{3}\end{array}\right]$ \\
\hline
\end{tabular}

\section{Robot position control}

An important issue for the guidance of a robot is the precise localization of the end effector in the intraoperative space. To do so, alternatively to conventional position encoders, localization modules are usually placed on the robot or its end-effector [19], [20]. Here, we also designed a specific localization device that can be use to compute the LPR position and orientation according to the CT/MR reference. This localization device is a square frame made of epoxy resin ensuring CT visibility, and is filled with oil for MR visibility (Figure 8). It is fastened with screws to the rotating base of the robot so that it is totally integrated into the needle holder architecture.

Each time we want to know the robot position to check if it is correct, we have to acquire a set of images which are automatically processed in order to detect the localization device and find 
the orientation of the robot and the entry point. These images also allow the radiologist to confirm the adequate progression of the procedure.

LPR localization involves image processing algorithms that have been developed and validated on CT images so far. The first step is a thresholding that leads to a binary image. A mathematical morphology closing operation comes next to fill in the holes of the image. Then, all the regions of the image are labelized in connected components, and a priori knowledge on the localization device is applied to conserve only the points of interest. The size of all connected objects is measured and only objects whose area is smaller than the area of the longest diagonal of the localization device are kept. Furthermore, since the localization device is made of bars, its slices in CT images are rectangular-shaped. We use then the ratio between the singular values of all object inertia ellipses in order to detect and keep only rectangle-shaped objects. These two treatments lead to images containing a large majority of localization device points for a minority of points of other objects (Figure 9).

A Chamfer 3-D matching algorithm [21] is then used in order to register the segmented cloud of points with a 3-D model of the localization device (Figure 10). As a result, the registration parameters (rotation, translation) give the position and orientation of the localization device, i.e., the position of the robot and of the needle, since the geometric relationships between all these elements are precisely known.

\section{Experimental results}

The first experiments on phantom have shown the capacity of LPR to reach its target with millimeter accuracy. Open-loop performance experiments, consisting in moving the robot to a particular position/attitude on a plane surface and checking its positioning accuracy, showed a strap- 
based translation accuracy of $5 \%$ of the displacement $d$ : for instance, if $d=20 \mathrm{~mm}$ then the maximum error is $1 \mathrm{~mm}$. Rotation and inclination accuracy were less than $1^{\circ}$.

Experiments on a foam rubber block in which we inserted a polyether-cetone disc with a $1 \mathrm{~cm}$ hole as a target consisted in trying to reach the target hole from an unknown position and orientation of the robot, with the control of CT images. On 6 punctures (2 vertical and 4 with an arbitrary orientation) starting from different initial positions, the target point was reached in all the cases with an error smaller than $2 \mathrm{~mm}$ (Figure 11).

Initial in-vivo animal experiments on 4 pigs have been conducted within a CT environment. The whole experimental setting involved the following steps:

- Initial injection of an artificial CT-visible target, simulating the tumour to be punctured, in the liver of a pig under general anaesthesia;

- Installation of the robot frame around the pig in the CT table, with the needle-holder part approximately located on the pig abdomen surface near the expected puncture entry point;

- Acquisition of a first set of CT images;

- CT images are used (1) by the radiologist to indicate the desired entry and target points, and (2) by the robot control software to segment and register the localization device and thus to determine the current robot position;

- Computation of the commands to send to the pneumatic actuators and automated realization of the robot motion;

- Acquisition of a new set of CT images, so that the radiologist can check, before the puncture, that the robot and needle have reached the desired position and orientation on the pig's abdomen surface;

- If necessary, these last 3 steps can be iterated in order to ensure optimal accuracy; 
- The radiologist determines then the needle penetration depth and gives the puncture movement order to the robot. Robot clamps can release the needle body once its tip has been inserted into the target.

These in-vivo experiments have demonstrated the feasibility of the LPR concept in clinical conditions, with resulting accuracies measured within the clinically acceptable range of 1 centimetre (Figure 12).

\section{Discussion and conclusion}

Translation accuracy of the robot has proved to be very satisfactory upon small distances (less than $30 \mathrm{~mm}$ ). Over this limit, the displacement is not as much accurate. Nevertheless, since the loop is closed by CT/MR image acquisition and supervised by the physician, it is possible to remain very accurate, as the final movement can always be set to be less than $30 \mathrm{~mm}$. In such conditions, experiments gave very promising results.

Segmentation and registration algorithms are currently based on image data acquired in a small volume that include the localization device, with post-motion processing. Further work will be done in order to provide, in real-time during robot motion, a fast closed control loop based on a single slice image acquisition.

Algorithms used during the LPR localization process have initially been developed with CT images. However, thanks to materials employed to construct the robot, to its compact architecture, and to the remote pneumatic energy source used to actuate it, LPR is fully MRI compatible. Thus, we are currently adapting the segmentation and registration algorithms for MR images. This will allow, shortly, first LPR experiments with MRI on healthy volunteers, and will further demonstrate the possibility with LPR to transform a simple CT or closed MR into a real-time controlled interventional imaging device. 


\section{REFERENCES}

[1] R. H. Taylor and D. Stoianovici, “Medical robotics in compter-integrated surgery,” IEEE Transactions on Robotics and Automation, vol. 19, no. 5, pp. 765-781, October 2003.

[2] K. Cleary, A. Melzer, V. Watson, G. Kronreif, and D. Stoianovici, "Interventional robotic systems: applications and technology state-of-the-art,” Minimally Invasive Therapy Allied Technologies, vol. 15, no. 2, pp. 101-113, April 2006

[3] J. F. Schenck, "The role of magnetic susceptibility in magnetic resonance imaging: MRI magnetic compatibility of the first and second kinds," Medical Physics, vol. 23, no. 6, pp. 815-850, June 1996.

[4] K. Chinzei, R. Kikinis, and A. Jolesz, "MR compatibility of mechatronic devices: Design criteria,” In Proceedings of the 2nd MICCAI, pp. 1020-1031, Cambridge, September 1999.

[5] K. Chinzei, K. Yoshinaka, and T. Washio, "Numerical Simulations and Lab Tests for Design of MRCompatible Robots," In proceedings of the IEEE International Conference on Robotics and Automation, pp.3819 - 24, 2006.

[6] R. Gassert and R. Moser and E. Burdet and H. Bleuler, "MRI/fMRI-Compatible robotic system with force feedback for interaction with human motion,” IEEE/ASME Transactions on Mechatronics, vol. 11, no. 2, April 2006.

[7] B. T. Larson, N. V. Tsekos, A.G. Erdman, E. Yacoub, P.V. Tsekos, and I.G. Koutlas, "Design of an MRICompatible Robotic Stereotactic Device for Minimally Invasive Interventions in the Breast,” Journal of Biomechanical Engineering, vol. 126, no. 4, pp. 458-465, August 2004.

[8] H. Fischer, S. Kutter, J. Vagner, A. Felden, S.O.R. Pfleiderer, W.A. Kaiser, "ROBITOM II : Robot for biopsy and therapy of the mamma," In proceedings of the IEEE International Conference on Systems, Man and Cybernetics, vol. 3, pp. 2530- 2534, October 2004.

[9] A. Krieger, R.C. Susil, C. Ménard, J.A. Coleman, G. Fichtinger, E. Atalar, and L.L. Whitcomb, “Design of a novel MRI compatible manipulator for image guided prostate interventions," IEEE Transactions on Biomedical Engineering, vol. 52, no. 2, pp. 306-313, February 2005.

[10] K. Chinzei, N. Hata, A.J olesz, and R. Kikinis, "Surgical Assist Robot for the Active Navigation in the Intraoperative MRI: Hardware Design Issues," In proceedings of the IEEE/RSJ International Conference on Intelligent Robots and Systems, vol. 1, pp. 727-732, Takamatsu, Japan, Oct 30-Nov 3, 2000.

[11] N. V. Tsekos, A. Özcan, and E. Christoforou, “A Prototype manipulator for magnetic resonance-guided interventions inside standard cylindrical magnetic resonance imaging scanners,” Journal of Biomechanical Engineering, vol. 127, no. 6, pp. 972-980, November 2005.

[12] A. Yamamoto, K. Ichiyanagi, T. Higuchi, H. Imamizu, R. Gassert, M. Ingold, L. Sache, and H. Bleuler, "Evaluation of MR-compatibility of electrostatic linear motor," In Proceeding of the IEEE International Conference on Robotics and Automation, pp. 3658-3663, Barcelona, Spain, April 2005.

[13] J. Vogan, A. Wingert, J. S. Plante, S. Dubowsky, M. Hafez, D. Kacher, and F. Jolesz, "Manipulation in MRI Devices using Electrostrictive Polymer Actuators: With an Application to Reconfigurable Imaging Coils," In Proceeding of the IEEE International Conference on Robotics and Automation, vol. 3, pp. 2498-2504, May 2004.

[14] A. V. Gonzales, J. Troccaz, Ph. Cinquin, A Guerraz, F. Pellisier, P. Thorel, B. Tondu, F. Courreges, G. Poisson, M. Althuser, and J-M. Ayoubi “Experiments with the TER Tele-echography Robot,” In proceedings of the 5th International Conference on Medical Image Computing and Computer-Assisted Intervention, vol. 2488, pp. 138-146, London, UK, 2002.

[15] P. Berkelman, P. Cinquin, J. Troccaz, J. Ayoubi, C. Letoublon, and F. Bouchard “A Compact, Compliant Laparoscopic Endoscope Manipulator,” In proceedings of the IEEE International Conference on Robotics and Automation, vol.2, pp. 1870-1875, Washington DC, May 2002.

[16] P. Cinquin, P. Berkelman, A. Jacquet, J. Arnault, "System for positioning on a patient an observation and/or intervention device,” - International patent BF n 02/05848, 13 May 2002.

[17] N. Zemiti, T. Ortmaier, and G.Morel, “A New Robot for Force Control in Minimally Invasive Surgery,” In proceedings of the IEEE/RSJ International Conference on Intelligent Robots and Systems, pp. 3643-3648, Sendai, Japan, September 2004.

[18] J. Hong, T. Dohi, M. Hashizume, K. Konishi, and N. Hata, “An ultrasound-driven needle-insertion robot for percutaneous cholecystostomy,” Physics in Medicine and Biology, vol. 49, no. 3, pp. 441-455, 2004.

[19] B. Maurin, O. Piccin, B. Bayle, J. Gangloff, M. de Mathelin, L. Soler, and A. Gangi, “A new robotic system for CT-guided percutaneous procedures with haptic feedback," In Proceedings of the Computer Assisted Radiology and Surgery Congress, pp. 515-520, Chicago, USA, June 2004. 
[20] R. C. Susil, J. H. Anderson, and R. H. Taylor, "A Single Image Registration Method for CT Guided Interventions," In Proceedings of the 2nd International Conference on Medical Image Computing and Computer-Assisted Intervention, Cambridge, England, LNCS 1679, vol.1679, pp. 798-808, September 1999.

[21] G. Borgefors, "Hierarchical chamfer matching: a parametric edge matching algorithm” IEEE Transactions on Pattern Analysis and Machine Intelligence, vol. 10, no. 6, pp. 849-865, November 1988. 


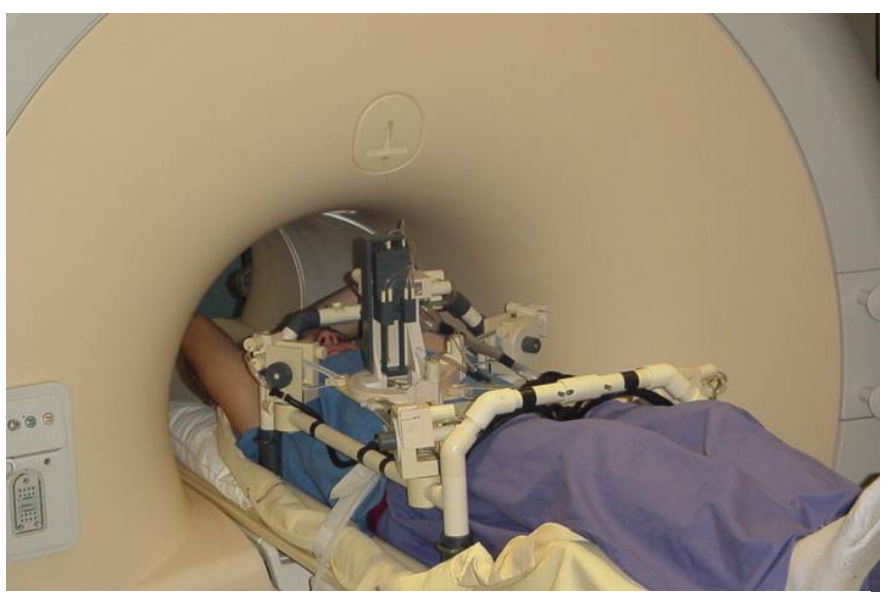

Figure 1: LPR on patient during MRI exam 


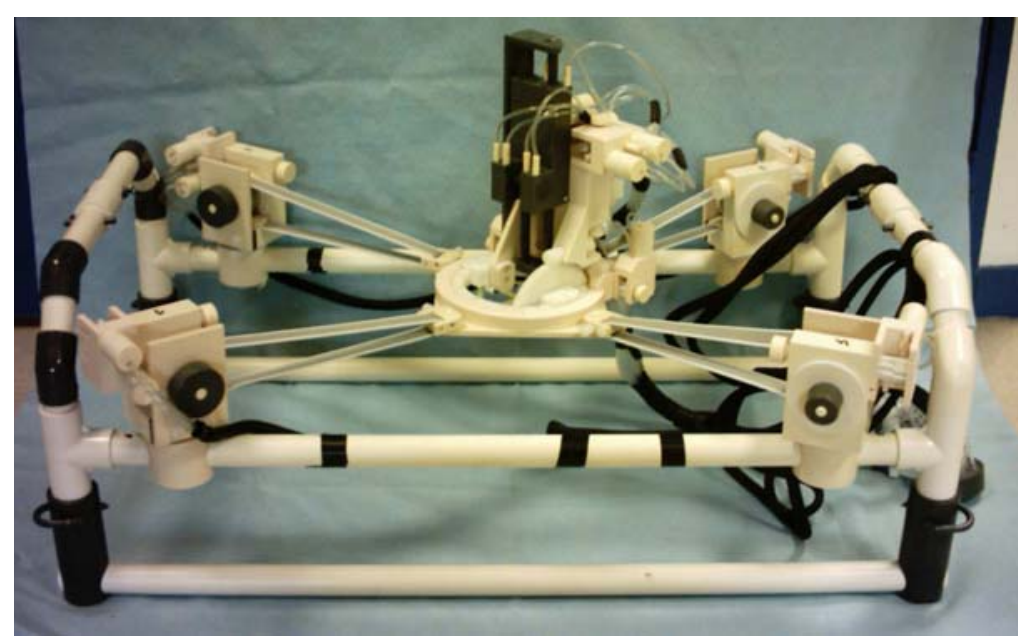

Figure 2: The LPR entire robot 


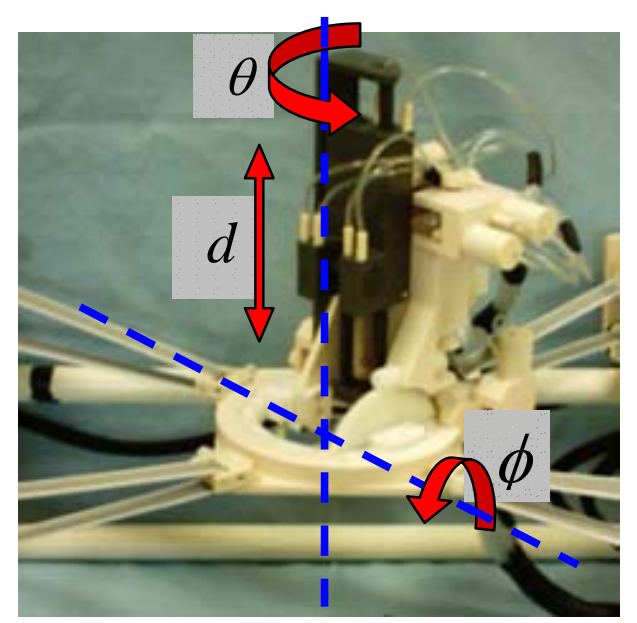

Figure 3: Degrees of Freedom of the needle holder 


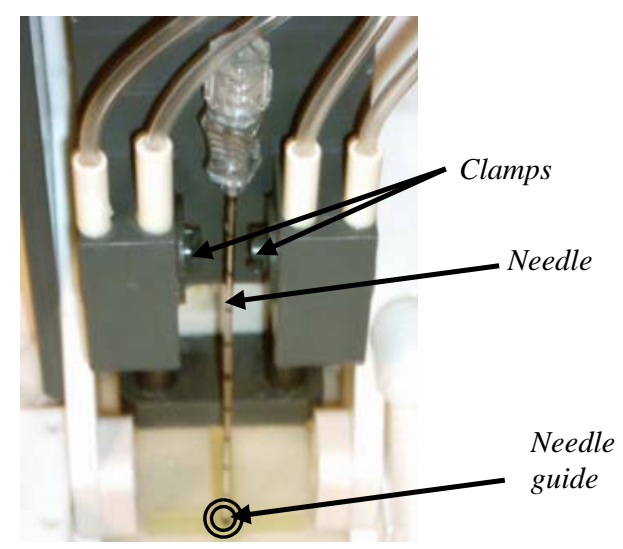

Figure 4: The needle holder puncture part 


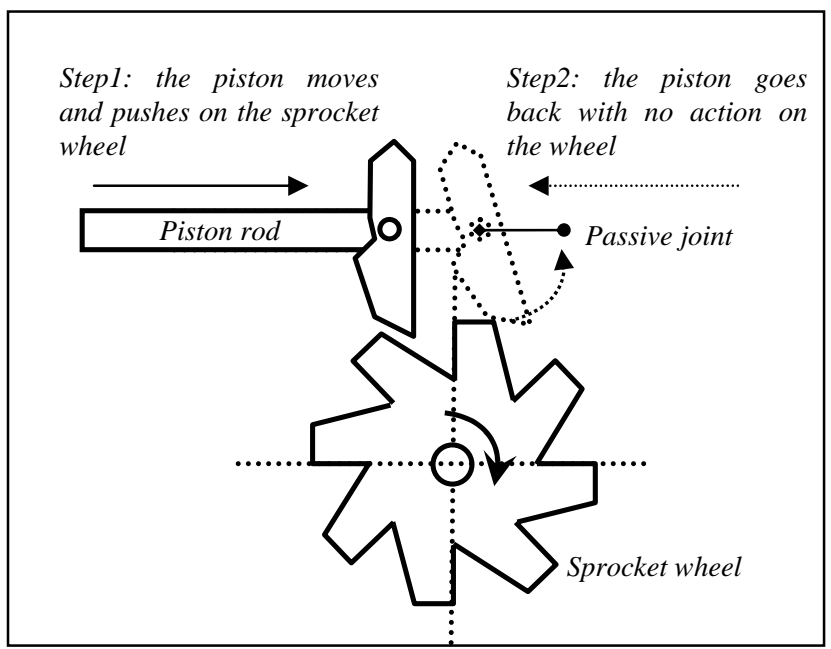

Figure 5: Principle of the pneumatic actuator for controlling the LPR movement 


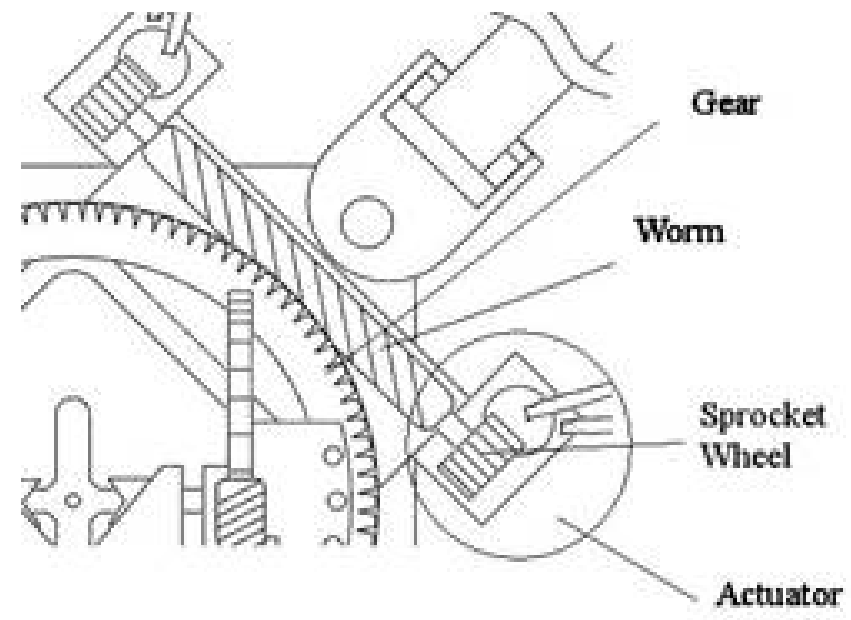

Figure 6: Needle holder rotation actuator 

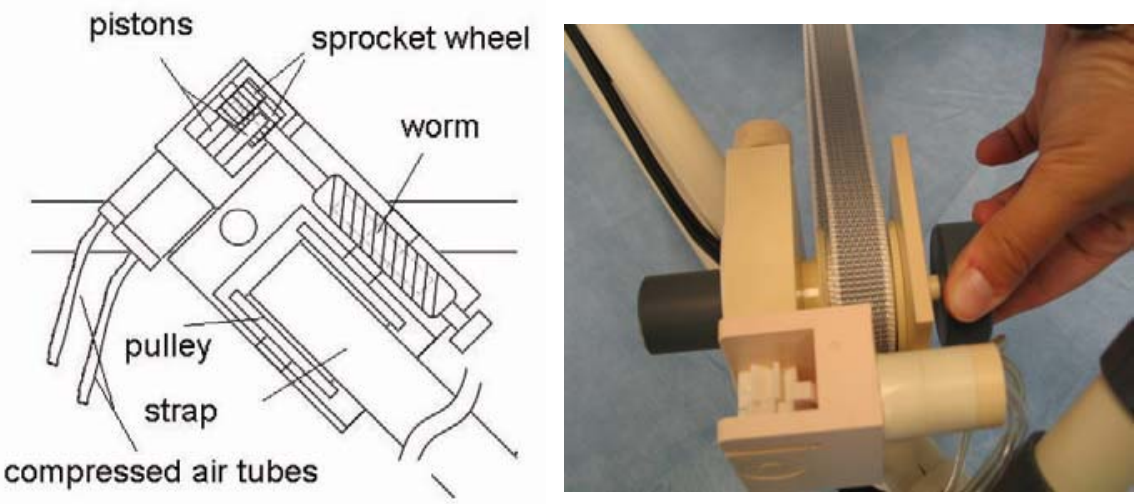

Figure 7: Principle of the translating actuator 


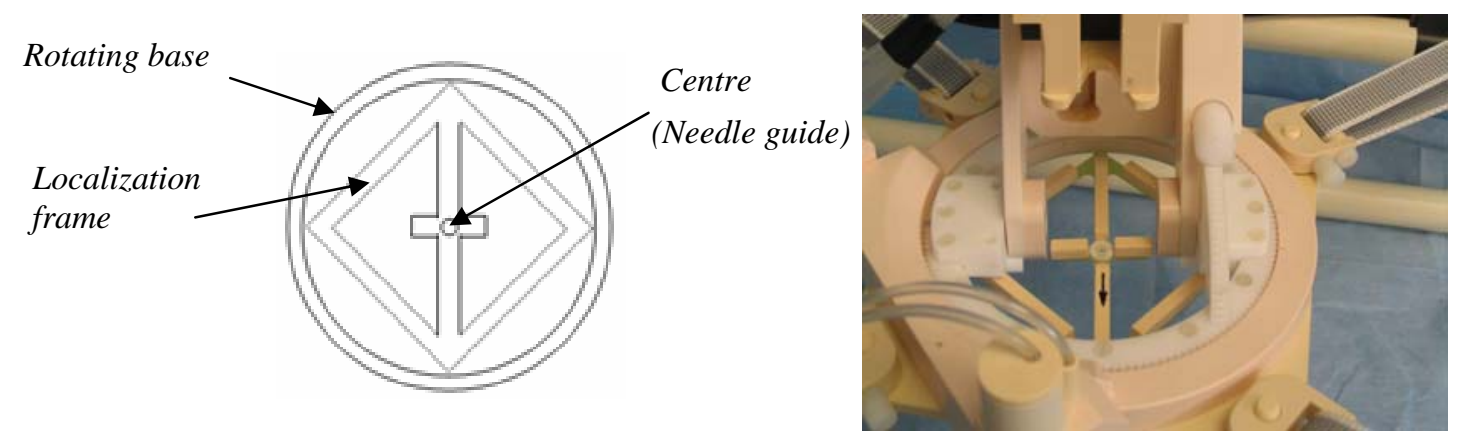

Figure 8: The localization device 

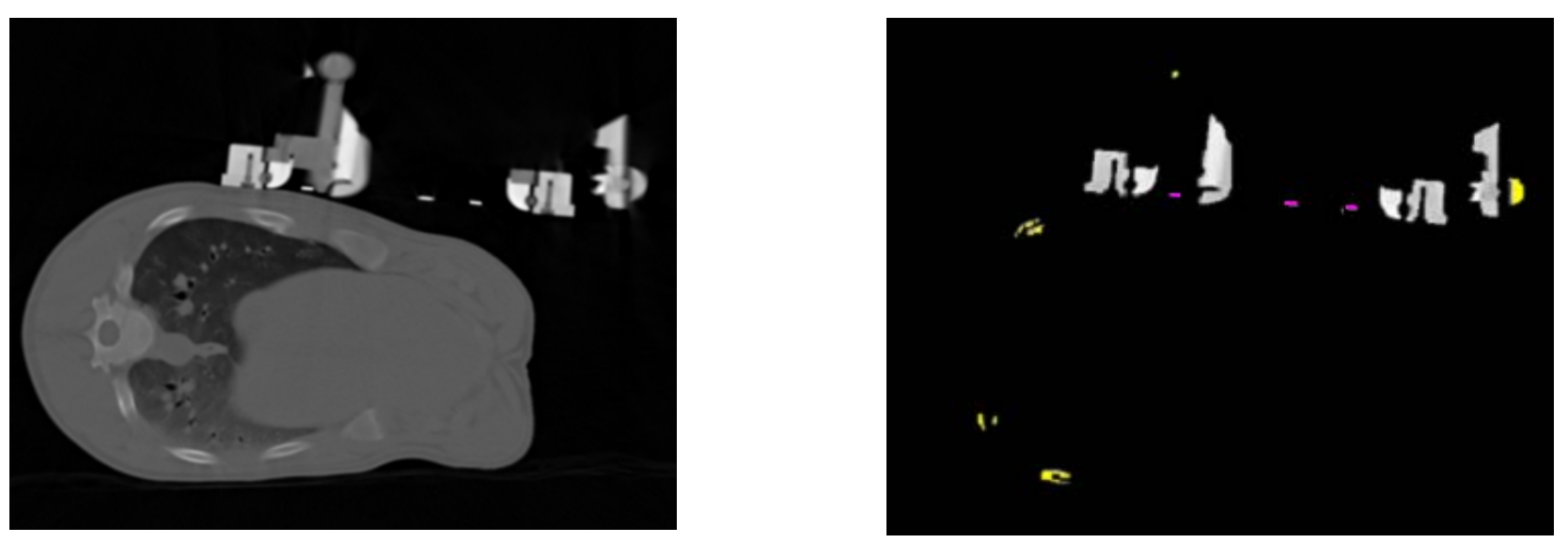

Figure 9: (a) CT image acquired during animal experiment. (b) The thresholding step extracts parts from the localization device or from the rest of the robot, and ribs. Geometric criteria succeed in selecting only parts from the localization device (in pink). 


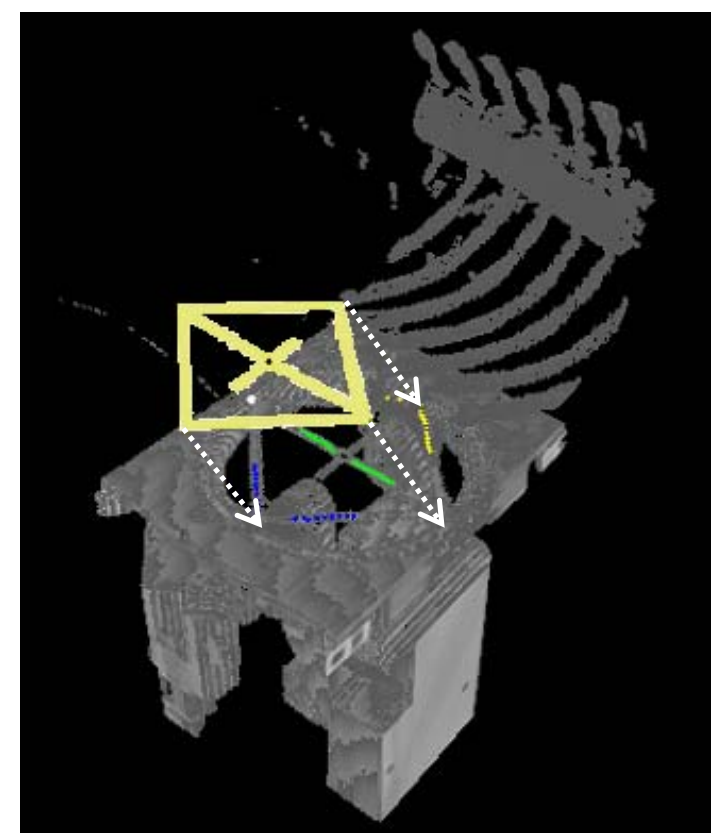

Figure 10: Schematic representation of the final matching step that registers the cloud of points extracted on CT images (colour points in this 3-D view of CT data) with a 3-D model of the localization device. 


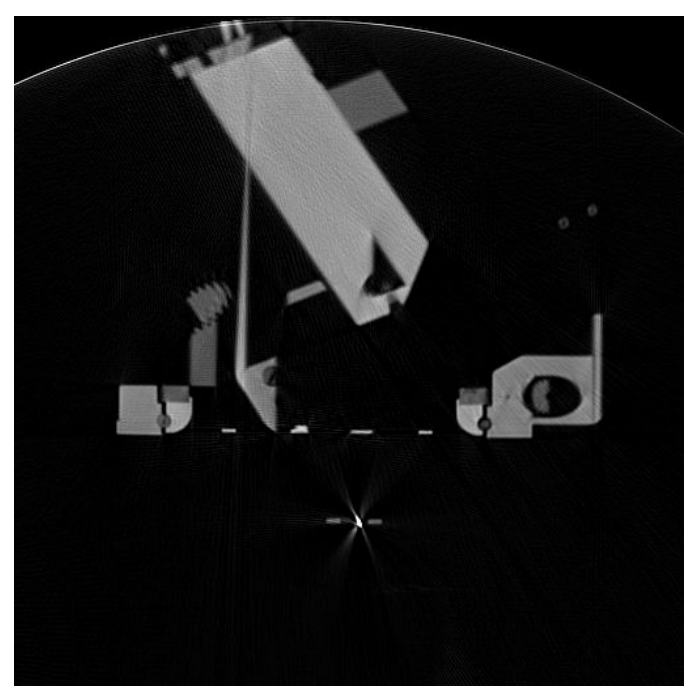

Figure 11: The needle has successfully reached the center of the target, following an oblique out-of-plane path. 


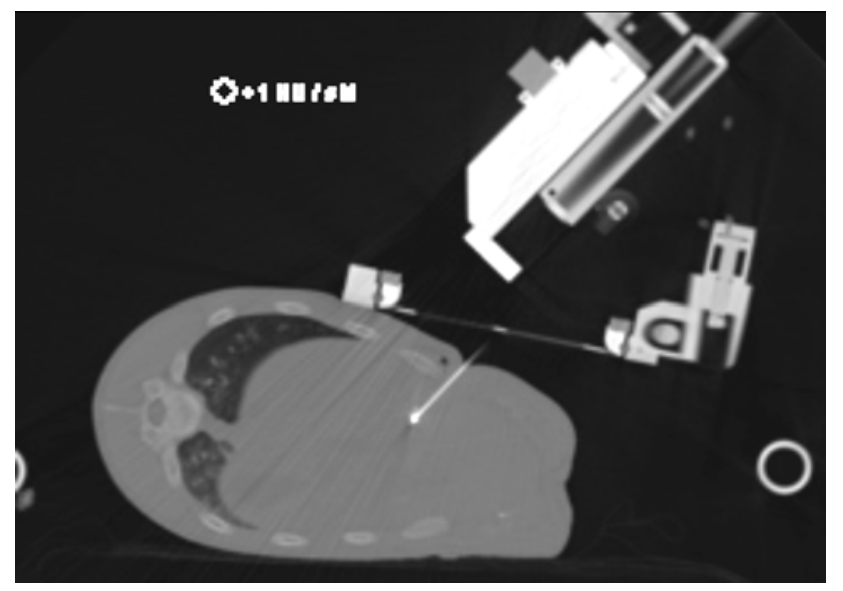

Figure 12: Successful in-vivo puncture of an artificially created target in a pig liver. 